\title{
A Deep Learning Based Strategy to Promote the Performance of Project Management
}

\author{
Author: Yo-Hsien Chen ${ }^{1}$; Shin-Jye Lee ${ }^{1}$; Ching-Hsun Tseng ${ }^{1}$
}

\author{
Affiliation: Institute of Management of Technology, National Chiao Tung University, \\ Taiwan $^{12}$;Department of Computer Science, The University of Manchester, Manchester, $\mathrm{UK}^{3}$
}

E-mail:squarea3008@gmail.com ${ }^{1}$;camhero@gmail.com² ${ }^{2}$ hank131415go61@gmail.com ${ }^{3}$

DOI: 10.26821/IJSHRE.9.3.2021.9306

\begin{abstract}
The project management refers to the task that has a starting point and ending point within an organization and a group of combining interrelated work. Accordingly, project management implies the use of management knowledge, tools, technology in project activities, and resources planned beforehand to solve the problems derived from projects or accidently requirements. Besides, Project management can be divided into four phases: planning, executing, monitoring, and closing. In these processes, there are some limitations, such as time, cost, manpower, resources, and etc. Traditionally, it may be the use of PERT (Program Evaluation and Review Technique), which refers to a flow chart that depicts the sequence of project activities and indicates the time of each activity or the associated costs. An effective project management must consider the execution of schedule on time, and identifies potential problems. Inasmuch as the rapid progression of AI technology in the industrial field, the application of AI technology in the technology management has become an increasing issue as an interdisciplinary study. Therefore, this work applies the deep learning method to obtain the approximated information of similar project processes for the reference of decision making in processing the project management, and it therefore brings a good effort to attain an effective project management in an intelligent manner.
\end{abstract}

\section{Keywords: Deep learning, Project management}

\section{INTRODUCTION}

For managers, project management has almost become an unscheduled task at work. When an organization needs to create something new, or when something needs to be reinvented, it is the most common opportunity for a project. Therefore, you may suddenly be assigned to set up a new website for the company, install a new machine for the factory, or set up a branch office in another city. These tasks that are not part of your daily work can be considered a project. A project contains the following qualities: Clear starting and ending points (with time limits), budget (with cost control). Clearly define the scope of work. And there are specific results. Project management refers to the activities of planning, scheduling, and control in order to achieve goals. In the field of project management, there is a very important concept, that is, the iron triangle: budget, time and scope. The content of the project is all-encompassing and varies in size. Regardless of the content of the project, the challenge for the responsible person is to complete the project within the established time and budget. Therefore, a person who can complete a project with a predetermined quality within a predetermined period of time is the highest level that many companies pursue. In general, the project management community is accustomed to understanding the project management with the structure of the "iron triangle". Each end of the triangle represents the limits of project management (respectively budget, time and scope), and the three are closely related. Any change in the end will have a positive or negative impact on the quality of the project. Some experts believe that speed, cost and quality, the three can only choose the second, that is: If you want the project to be fast and cheap, the quality cannot be too good. If you want the project to be good and cheap, then the speed will not be too fast. If you want the project to be fast and good, the price is definitely not cheap. Of course, some people disagree with this statement. In fact, management can make a big opportunity to make the project fast, good and cheap, and the key is to carefully evaluate and plan the various stages of project management, and integrate the skills of each knowledge field into each stage. . 
Volume 9 Issue 3 March 2021

Complete the phase objectives in a step-by-step manner.

Artificial intelligence refers to the wisdom exhibited by machines made by humans. It replaces the labor in the factory with a smarter robotic arm in the early days, filters the spam by machine learning, analyzes our behavior and delivers related advertisements. Due to the breakthrough of deep learning technology, unmanned vehicles are about to replace drivers, and even white-collar work with brain power is in jeopardy. The machine learning which we often hear is part of artificial intelligence[1], and deep learning is another kind of machine learning. Machine learning, as the name implies, machine learning is to let the machine (computer) have the ability to learn like human beings. To understand machine learning, we should first look back at the process of human learning, which can be roughly divided into two steps of training and prediction. In order for a machine to have the ability to learn and judge like a human being, it is necessary to transfer the process of learning and judging the human brain to the machine. Basically, it is to use data for training and prediction, including four steps: acquiring data, analyzing data, and building a model. Forecasting the future: When the learning is completed, the new data can be imported into the model to predict the future. For example, if it rains and the temperature and humidity reach the standard, you can predict that the rainbow will be seen in the opposite direction to the

sun.

Machine learning is similar to the process of human learning. It is necessary to classify before analyzing and understanding, and finally taking action. The process of classification is actually a kind of right and wrong. For example, this photo is a cat or a non-cat. Machine learning is a computer-executed program that learns itself and processes the large amount of data while learning the classification method. Just as human beings are learning, machine learning is processing data.

Artificial neural network, also known as neural network, is a mathematical model that mimics the structure and function of biological neural networks. It is used to evaluate or approximate functions. It is the most commonly used model of artificial intelligence. Scientists mimic the neural network of the human brain to explain the neuroscience theory of brain neuron changes during learning. Synaptic stimulation of a neuron to a neuron under the synapse can lead to an increase in synaptic transmission efficiency. That is, the weight on the artificial neural network. Deep learning is to let the computer analyze the data to find the feature value, instead of the human to determine the feature value, just as the computer can have deep learning. While deep learning uses a multi-layer neural network, the more hidden layers in theory, the higher the degree of freedom and precision, but the opposite is true, because error backpropagation is difficult to pass back to the higher-level neurons, as if the supervisor was provided by the employee. The information is judged. The correct information is that the next employee provides back-propagation to the supervisor, and the correction is carried out by the supervisor to the employee. When the class is too much, the effect is not good. Therefore, deep learning not only uses a multi-layer neural network, but also uses an automatic encoder for unsupervised learning.

\section{RELATED WORK}

After experiencing the Internet sweeping global physical retail, technology has driven the next wave of industrial transformation, and the demand for new technology talents has also emerged. Just as technology such as the Internet of Things has gradually changed human life, in the operation of enterprises, big data, AI and other technologies have also entered the industry from the Internet, helping brands to understand the contours of customers, and invisibly changing the processes and thinking of traditional enterprise operations. More and more industries are aware of the importance of engineers and even selforganizing IT teams within thecompany.

This wave of new technology talents is hot, and with the penetration of big data, cloud, artificial intelligence and other technologies into all walks of life, demand continues to be bullish. In 2024, the demand for scientific and technological talents in the United States will grow by $12 \%$. The main areas are data analysis, health technology, technology security, web development, project management and database management.

The work of replacing new technology seems to be more and more, but in the end, there will be a new type of work that needs to be handled by "people." According to McKinsey's global report, $20 \%$ of the world's workers, $70 \%$ of their work can be automated, and after the work is automatically replaced, the remaining $30 \%$ of the work will be assembled to create new type of work content.

Taking the application of machine learning as an example, the new work will abandon the routine and turn it into something that the robot can't do. In order 
Volume 9 Issue 3 March 2021

to achieve higher efficiency, the acceleration of technology is not only online, but human beings are transferred to the role of managers, dealing with exceptions to program operations, more complex jobs or innovative service ideas. The global industrial environment is constantly changing[1], and enterprises must be able to respond quickly to changes, and fixed jobs can no longer meet demand. Traditional work is clearly distinguished between a certain department and a certain unit. Even if the organization will carry out manpower transfer every time, but as the company seeks to improve the internal operation efficiency, the workers are no longer restricted to perform fixed work projects, but are more flexible local projectorientation.

A project-oriented approach can increase the overall productivity of the company. And through the establishment of a network work platform by internal technical staff, employees can arrange work affairs on the platform through project. In this form, workers jump out of their respective professional title frameworks, adjust job details with projectgoals, and create better work efficiency.

\section{METHOD}

Deep learning is a branch of machine learning. It is the fastest growing field in artificial intelligence. Deep learning simulates the operation of human neural networks. Common deep learning architectures such as Multilayer Perceptron, Deep Neural Network, Convolutional Neural Network, Recurrent Neural Network. Deep learning is especially applied to natural language processing, biomedical and other fields, and has achieved very good results. As early as the 1960s and 1970s, scientists proposed various machine learning algorithms. However, limited by the computing power of the computer at the time, and the acquisition of a large amount of data is not easy, machine learning has not been very successful. A large amount of data is generated as devices, machines, and systems are connected globally. Coupled with the development of decentralized storage such as Hadoop, NoSQL, etc., a large amount of information is provided, and a large number of parallel operations of the server, such as Spark, provide a huge amount of computing energy. A large amount of data and computing power is like fuel, which promotes the accelerated development of machine learning and deep learning. A deep neural network is a neural network with at least three hidden layers. Similar to shallow neural networks, deep neural networks can also model complex nonlinear systems, but the extra levels provide a higher level of abstraction for the model,thus improving the model's capabilities.
Deep Neural Networks is a discriminant model that can be trained using backpropagation algorithms. The weight update can be solved by the stochastic gradient descent method using the following formula:

$$
\Delta w_{i j}(t+1)=\Delta w_{i j}(t)+\eta \frac{\partial C}{\partial w_{i j}}
$$

Among them, $\eta$ is the learning rate, and $\mathrm{C}$ is the cost function. The choice of this function is related to the type of learning likesupervised learning, unsupervised learning, enhanced learning) and enabling functions. For example, to conduct supervised learning on a multi-classification problem, the usual choice is to use ReLU as an enable function and cross-entropy as a cost function.

In this paper, we will use the elements that will be used in the scope of project management, such as time, cost, quality, etc., as the eigenvalues, and as the input value of the model, whether the project stroke that is discharged can be used as a label value. And the output value to achieve the purpose ofthis paper.

\section{CONCLUSION}

Since AI will have an impact on business strategy and human hiring, many project management managers have to play an important role in leading companies to prepare. From 2010 to 2015, the company's investment in artificial intelligence has increased by nearly 7 times; before 2021, 30\% of the company's new profits will come from business solutions based on artificial intelligence technology. The impact of artificial intelligence on the business will depend on its industry, business, organization and customers. As artificial intelligence and machine learning continue to deliver economic benefits, many of the areas of work now dominated by professionals will become general lowcost jobs. The impact of artificial intelligence on various industries will prompt companies to adjust their business strategies. Because artificial intelligence can transform complex work into measurement services, many highly competitive, high-profit industries become paid for by companies like hydropower. Artificial intelligence will eventually replace many of the routines in the IT department, with operational aspects such as systems management, service desk, project management and application support being most likely to be affected. Although the application of artificial intelligence will lead to the disappearance of some jobs, it can also improve some of the skills shortages, and the IT department will be able to focus more on more creative work to create corporate differentiation. In the end, artificial 
intelligence and humans will be separated by themselves. Artificial intelligence excels at solving problems that are well-defined and narrow-scoped, while humans are good at defining problems that need to be solved and solving complex problems. Because humans can use all kinds of knowledge and skills, and try to solve problems in different ways. In addition, humans can cooperate with each other and adjust to the situation immediately when there is a major change in the situation.

\section{REFERENCE}

[1] Lee, S. -J. \#, Lin, G. T. -R. and Hsi, P. -H. (2017). Industrial Cluster Development and Its Contribution to Economic Growth in Taiwan - Hsinchu Science and Industrial Park. Journal of Scientific \& Industrial Research, vol. 76, no. 5, pp. 273-278. $(1$ st Author, SCI Impact Factor $=0.557)$ 